\title{
Influence of extracellular nanovesicles derived from adipose-derived stem cells on nucleus pulposus cell from patients with intervertebral disc degeneration
}

\author{
ZHIQIANG ZHANG, LIANG ZHANG, JIANDONG YANG, JIJUN HUANG, \\ JUN CAI, SHENGFEI ZHANG, XINMIN FENG and QIANG WANG \\ Department of Orthopedics, Clinical Medical College of Yangzhou University, Orthopaedic Institute, \\ Northern Jiangsu People's Hospital, Yangzhou, Jiangsu 225001, P.R. China \\ Received February 15, 2019; Accepted January 26, 2021
}

DOI: $10.3892 /$ etm.2021.10866

\begin{abstract}
An increasing number of individuals are suffering from lower back and neck pain caused by intervertebral disc degeneration each year. Although the application of mesenchymal stem cells (MSCs) has provided desirable results in the treatment of intervertebral disc degeneration, there are multiple risks associated with the directed application of MSCs. An increasing number of studies have suggested that stem cells, through the release of extracellular nanovesicles, have vital functions in tissue regeneration and repair with low risk. The present study investigated the effect of extracellular nanovesicles derived from adipose-derived stem cells (ADSCs) on nucleus pulposus (NP) cells from patients with intervertebral disc degeneration. Human NP cells were obtained from patients with intervertebral disc degeneration undergoing surgical procedures in addition to ADSCs from liposuction patients. ADSC-derived extracellular nanovesicles were isolated and characterized. The differentiation and biological activity of NP cells cultured with or without ADSC-derived extracellular nanovesicles were assessed and inflammatory factors and intervertebral disc degeneration-associated markers were also measured. The results indicated that extracellular nanovesicles derived from ADSCs increased the migration and proliferation of NP cells and inhibited inflammatory activity, suggesting their utility for the treatment of intervertebral disc degeneration.
\end{abstract}

\section{Introduction}

Intervertebral disc degeneration is a debilitating condition of the joints that affects millions of individuals and remains an

Correspondence to: Professor Qiang Wang, Department of Orthopedics, Clinical Medical College of Yangzhou University, Orthopaedic Institute, Northern Jiangsu People's Hospital, 98 Western Nantong Road, Yangzhou, Jiangsu 225001, P.R. China E-mail: qiangwang0525@126.com

Key words: intervertebral disc degeneration, adipose-derived stem cells, nucleus pulposus cell, exosomes, anti-inflammatory unresolved problem (1). Intervertebral disc degeneration presents a global public health and economic burden, causing pain in the back or neck and frequently in the arms and legs, leading to increased morbidity and disability (2). It is reported that $\sim 5.9$ million people are affected by intervertebral disc degeneration (3) and 1-2\% of the adult population becomes disabled secondarily to back pain in the USA per year (4). Intervertebral disc degeneration is thought to be mainly characterized by degenerative spinal changes and the gradual formation of osteophytes, disc narrowing and spinal stenosis (5).

Conservative therapies for intervertebral disc degeneration include non-invasive therapies, such as physical therapy, weight reduction and anti-inflammatory medication, or corticosteroid injection; the non-invasive treatments frequently fail and further result in chronic lower back pain (6). Surgical interventions include lumbar spinal fusion surgery and artificial disc replacement surgery (7).

Due to their ease of harvesting, chondrogenic differentiation capacity and high proliferation ability, as well as their paracrine and immune-modulating effects through the production of cytokines and growth factors, mesenchymal stem cells (MSCs) represent a promising option as a regenerative medical treatment that may potentially replenish defective tissue and regenerate healthy tissue in the degenerated intervertebral disc $(8,9)$. Yang et al (10) indicated that bone marrow-derived stem cells (BMSCs) were able to arrest the degeneration of the murine notochordal nucleus pulposus (NP) and contribute to the augmentation of the extracellular matrix in the NP by both autonomous differentiation and stimulatory action on endogenous cells. Centeno et al (11) reported that patients with degenerative disc disease treated with autologous cultured MSCs for lower back pain with radicular symptoms demonstrated significant improvements in pain and function, as well as overall subjective improvement with only minor adverse events through 6 years of follow-up.

However, studies have reported a series of risks that limit the direct application of MSCs in tissue repair and tissue regeneration. The intrinsic risk factors include cellular rejection and immunosuppression, infusion toxicity and the risk of iatrogenic tumor formation, and the extrinsic risks caused by human handling, such as culture conditions, cryopreservation and 
various other cell manipulations, remain a big challenge (12). In addition, the ossification of neo-cartilage differentiated from stem cells, as well as abnormalities in the proliferation and differentiation ability and cell phenotype (undesired hypertrophy), remain unresolved (13).

It was demonstrated that the repair and regeneration potency of MSCs in injured tissues or organs may not be attributed to their differentiation ability but rather to their paracrine signaling effects (14). Evidence has indicated that extracellular nanovesicles $(40-100 \mathrm{~nm}$ in diameter) released from cells carry microRNAs (miRNAs), mRNAs and proteins that mediate cell-cell microcommunication and the transport of paracrine factors, which may have a significant role during tissue repair and tissue regeneration, as well as immune regulation, leading to a therapeutic effect (15).

However, the effect of adipose-derived stem cell (ADSC)-derived extracellular nanovesicles on intervertebral disc degeneration has remained largely unexplored. The present study aimed to evaluate the effectiveness of extracellular nanovesicles isolated from ADSCs on NP cells from patients with intervertebral disc degeneration. It was indicated that ADSC-derived extracellular nanovesicles were able to stimulate chondrocyte migration and proliferation, and downregulate intervertebral disc degeneration-related inflammatory cytokines, indicating their potential therapeutic utility in intervertebral disc degeneration.

\section{Materials and methods}

Cell isolation and culture. The ADSCs were harvested from patients undergoing liposuction surgery for abdominoplasty (patients in good condition without hypertension, hyperlipidemia, diabetes and coronary heart disease; 3 male and 3 female patients; age range, 40-60 years). In total, 2,000-4,000 $\mathrm{ml}$ was taken from each patient, and the samples from all patients were not pooled together. ADSCs were isolated by digestion and centrifugation at $300 \mathrm{x} \mathrm{g}$, and were re-suspended and cultured in DMEM (Gibco; Thermo Fisher Scientific, Inc.) containing $10 \%$ (v/v) FBS (Hyclone; GE Healthcare Life Sciences) in $100-\mathrm{mm}^{2}$ tissue culture flasks at $37^{\circ} \mathrm{C}$ in a humidified incubator with $5 \% \mathrm{CO}_{2}(16)$. After $24 \mathrm{~h}$, the non-adherent cells were removed. After 10-14 days, adherent cells were trypsinized and subcultured.

NP cells were harvested from intervertebral disc degeneration patients undergoing lumbar spinal fusion surgery to relieve lower back pain (patients in good condition without hypertension, hyperlipidemia, diabetes and coronary heart disease; 3 male and 3 female patients; age range, 40-60 years) and the tissues were treated with $0.1 \%$ collagenase (Sigma-Aldrich; Merck KGaA) and $2 \mathrm{U} / \mathrm{ml}$ hyaluronidase (Sigma-Aldrich; Merck $\mathrm{KGaA}$ ) for $6-8 \mathrm{~h}$ in the incubator with $5 \% \mathrm{CO}_{2}$ at $37^{\circ} \mathrm{C}$. Next, the digested tissues were passed through a $200-\mu \mathrm{m}$ filter to remove any undigested particles. The suspension was centrifuged at $1,000 \times \mathrm{g}$ for $5 \mathrm{~min}$ at $4^{\circ} \mathrm{C}$, washed three times with sterile PBS and cultured in DMEM with $10 \%$ FBS and antibiotics (1\% penicillin/streptomycin) in the incubator with $5 \% \mathrm{CO}_{2}$ at $37^{\circ} \mathrm{C}$. When confluent, the NP cells were harvested using $0.25 \%$ trypsin-EDTA (Gibco; Thermo Fisher Scientific, Inc.) and re-seeded onto $10-\mathrm{cm}^{2}$ culture plates at a density of $1 \times 10^{6}$ cells per plate. The complete medium was replenished every other day (17). ADSCs and NP cells at passage 2 were used in the present study.

Extracellular nanovesicle isolation and identification. ADSC-derived extracellular nanovesicles were isolated as described previously (18). In brief, when ADSCs at passage 2 reached $75 \%$ confluence, they were cultured in $\alpha-M E M(G i b c o$; Thermo Fisher Scientific, Inc.) with extracellular nanovesicle-depleted FBS for $48 \mathrm{~h}$, and extracellular vesicle-depleted FBS was also used in the subsequent functional assays. Following the incubation, the supernatant was obtained and centrifuged at $500 \mathrm{x} \mathrm{g}$ for $10 \mathrm{~min}$ twice, at 2,000 $\mathrm{x} \mathrm{g}$ for 15 min twice, and at $10,000 \times \mathrm{g}$ for $30 \mathrm{~min}$ twice, all at $4^{\circ} \mathrm{C}$. The supernatant was then transferred to Ultra-Clear tubes (Beckman Coulter, Inc.) and centrifuged at 70,000 x g for $1 \mathrm{~h}$ at $4^{\circ} \mathrm{C}$. The pellet containing extracellular nanovesicles was washed with PBS and centrifuged at $70,000 \times \mathrm{g}$ for $1 \mathrm{~h}$ at $4^{\circ} \mathrm{C}$. The pellet was then carefully resuspended in $200 \mu \mathrm{l}$ PBS and used immediately or stored at $-80^{\circ} \mathrm{C}$.

The ultrastructure of the ADSC-derived extracellular nanovesicles was analyzed using transmission electron microscopy. ADSC-derived extracellular nanovesicle pellets were transferred to carbon-coated 200-mesh copper electron microscopy grids and incubated for $10 \mathrm{~min}$ at room temperature. Subsequently, the extracellular nanovesicles were stained with uranyl acetate and washed with PBS three times. After drying at room temperature, they were observed under a transmission electron microscope (19).

Particle size distributions were measured via NanoSight analysis. The concentration and size distribution of ADSC-derived extracellular nanovesicles were measured using tunable resistive pulse sensing analysis with qNano (Izon Science, Ltd.). ADSC-derived extracellular nanovesicles were placed in the Nanopore (NP150; cat. no. A37355; Izon Science, Ltd.) at a 47.0-mm stretch with a voltage of $0.6 \mathrm{~V}$. Izon Control Suite software v.2.2 (Izon Science, Ltd.) was used for data analysis.

Cell proliferation and migration. A Cell Counting Kit-8 (CCK-8; Dojindo Molecular Technologies, Inc.) was used to evaluate NP cell proliferation. In brief, $10^{3} \mathrm{NP}$ cells in $100 \mu \mathrm{l}$ were seeded into each well of a 96-well plate. After $12 \mathrm{~h}$, $50 \mu \mathrm{g} / \mathrm{ml}$ extracellular nanovesicles were added to the wells in the experimental group, while no extracellular nanovesicles were added to the wells of the control group. The medium was replaced every 2 days using fresh medium with or without extracellular nanovesicles and medium with extracellular vesicle-depleted FBS was used in the functional assays. At different time-points (1, 3, 5 and 7 days), $10 \mu \mathrm{l}$ of CCK-8 solution was added to each well, along with $90 \mu \mathrm{l}$ of high-glucose DMEM without FBS (Gibco; Thermo Fisher Scientific, Inc.). After another $3 \mathrm{~h}$ of incubation, the absorbance was measured spectrophotometrically at a wavelength of $450 \mathrm{~nm}$ with a microplate reader.

Scratch wound assays were used to evaluate the effects of extracellular nanovesicles on NP-cell migration, as described previously (20). In brief, $2 \times 10^{5}$ cells/well were seeded into 6 -well plates and incubated for $12 \mathrm{~h}$. A sterile $200-\mu \mathrm{l}$ pipette tip was used to make a scratch when the cells were $80 \%$ confluent and non-adherent cells were removed by washing 

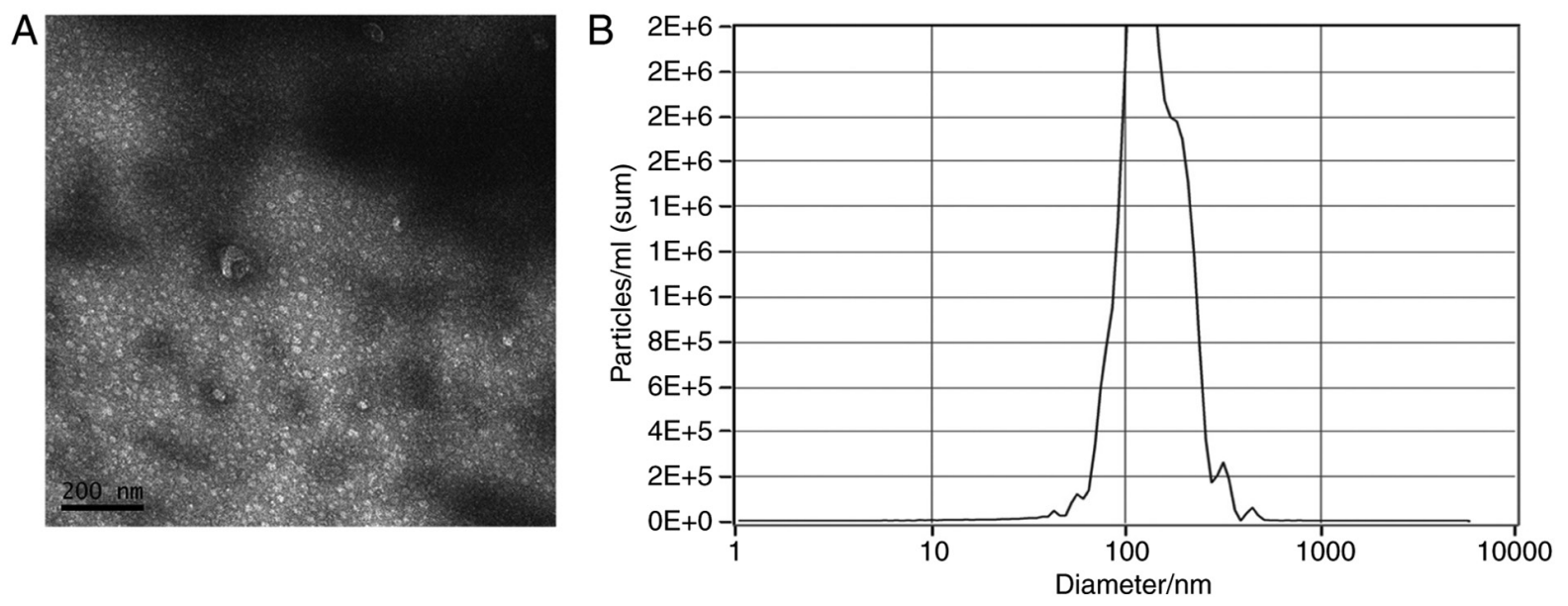

Figure 1. Characterization of exosomes isolated from the mesenchymal stem cell. (A) Transmission electron microscopy indicated that the size of the exosomes ranged from 40 to $200 \mathrm{~nm}$ (scale bar, $200 \mathrm{~nm}$ ). (B) NanoSight analysis indicated that the peak of the distribution of the diameters of the exosomes was $120 \mathrm{~nm}$.

with PBS. The cells were cultured with or without $50 \mu \mathrm{g} / \mathrm{ml}$ extracellular nanovesicles. Images were acquired at 0,12 and 24 h, and Image-Pro Plus 6.0 software (Media Cybernetics) was used to measure the scratched areas.

Chondrocytic gene expression. The total RNA was extracted from each specimen using TRIzol ${ }^{\circledR}$ reagent and complementary DNA was obtained by reverse transcription (RT) using the SuperScript First-Strand Synthesis system (Thermo Fisher Scientific, Inc.) for PCR. RT-quantitative PCR was performed to evaluate the expression levels of collagen-II (forward primer, 5'-GAGCCAAAGGATCTGCTGGT-3') and reverse primer, 5'-TTGGGGCCTTGTTCACCTTT-3'), aggrecan (forward primer, 5'-AAAAGGAGGCCACAGTGC TT-3' and reverse primer, 5'-GGCCGTACCAATCTCACA CA-3') and Sox-9 (forward primer, 5'-AGGAGAACCCCA AGATGCAC-3' and reverse primer, 5'-GAGGCGTTTTGC TTCGTCAA-3'). The expression of GAPDH (forward primer, 5'-GAGAAGGCTGGGGCTCATTT-3' and reverse primer, 5'-AGTGATGGCATGGACTGTGG-3) was quantified as an internal control. The PCR was performed using SYBR Green PCR master mix (Thermo Fisher Scientific, Inc.) and an ABI Prism 7000 Sequence Detection system with ABI Prism 7000 software (Applied Biosystems; Thermo Fisher Scientific, Inc.). The PCR thermocycling conditions were as follows: $95^{\circ} \mathrm{C}$ for $15 \mathrm{~min} ; 35$ cycles of $94^{\circ} \mathrm{C}$ for $1 \mathrm{~min}, 59^{\circ} \mathrm{C}$ for $1 \mathrm{~min}$ and $72^{\circ} \mathrm{C}$ for $1 \mathrm{~min}$; and $72^{\circ} \mathrm{C}$ for $10 \mathrm{~min}$. The specificity of the amplification of the expected DNA fragments was confirmed using $2 \%$ agarose gel electrophoresis and by analysis of the melting curves. An amplification reaction control with no RT enzyme was performed in order to assess the interference of potential genomic DNA in the RNA solution. The relative gene expression was calculated using the $2^{-\Delta \Delta \mathrm{Cq}}$ method (21).

Telomerase activity detected by PCR-ELISA. Telomerase PCR-ELISA (Thermo Fisher Scientific, Inc.) was used to determine the telomerase activity following the manufacturer's protocols and as described previously (22). After 7 days of incubation, the NP cells cultured with or without extracellular nanovesicles were digested, collected and homogenized in a lysis buffer, and centrifuged at $100 \mathrm{x}$ g for $10 \mathrm{~min}$ at $4^{\circ} \mathrm{C}$. The extracts were sent to an internal department for telomeric repeat amplification. Subsequently, the elongated fragments were amplified by PCR and the PCR products were quantified by ELISA, as previously described (22). The results were normalized to those obtained for a standard.

Levels of inflammatory cytokines. ELISAs were performed to evaluate the expression levels of inflammatory cytokines in the two groups. Media were collected and centrifuged at $168 \mathrm{x} \mathrm{g}$ for $5 \mathrm{~min}$ at $4^{\circ} \mathrm{C}$ to remove cellular debris, and subsequently, IL-1 $\alpha$ (cat. no. SLA50, R\&D Systems), IL-1 $\beta$ (cat. no. SLB50, R\&D), IL-6 (cat. no. S6050, R\&D), IL-17 (cat. no. S1700, R\&D), NF- $\kappa$ B-p65 (cat. no. ab176648, Abcam) and TNF- $\alpha$ (cat. no. STA00D, R\&D) in the supernatant were measured using Quantikine ELISA kits according to the manufacturer's protocols.

Statistical analysis. Cell proliferation, gene expression and protein levels are presented as the mean \pm standard deviation and were analyzed by one-way ANOVA followed by Tukey's post-hoc test. Statistical analysis was performed using GraphPad Prism version 5.0 (GraphPad Software, Inc.). $\mathrm{P}<0.05$ was considered to indicate statistical significance.

\section{Results}

Characteristics of exosomes. Observation by transmission electron microscopy indicated that ADSC-derived extracellular nanovesicles were characterized by diameters in the range of 40-200 nm and most of them presented with a round shape (Fig. 1A). It was observed by NanoSight analysis that the peak of the distribution of diameters of extracellular nanovesicles was $120 \mathrm{~nm}$ (Fig. 1B).

Effects of ADSC-derived extracellular nanovesicles on the proliferation and migration of human NP cells. As presented in Fig. 2A and B, the migration of NP cells cultured in the presence of ADSC-extracellular nanovesicles in the experimental group was increased compared with that of the control group $(\mathrm{P}<0.05)$. It was also indicated that human NP-cell 
A
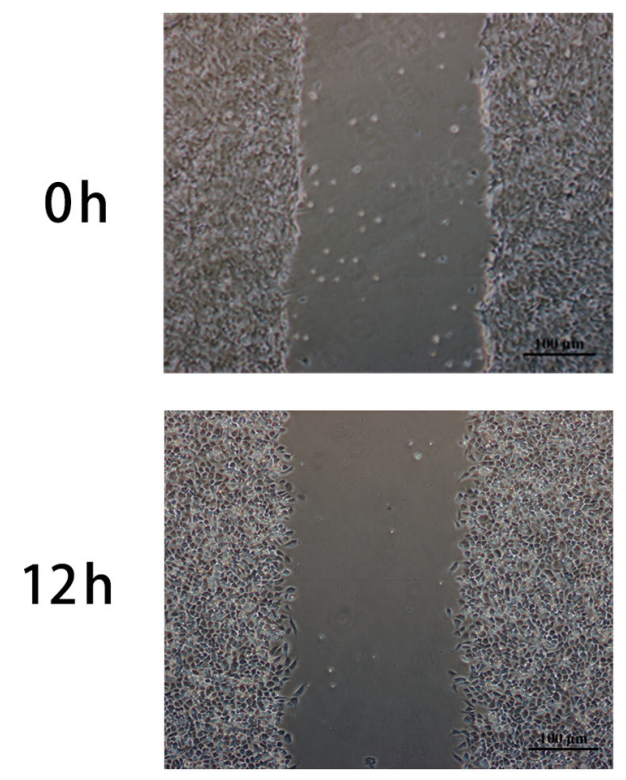

$24 \mathrm{~h}$

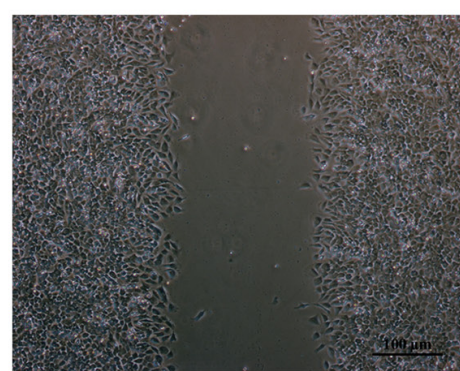

Expe. group
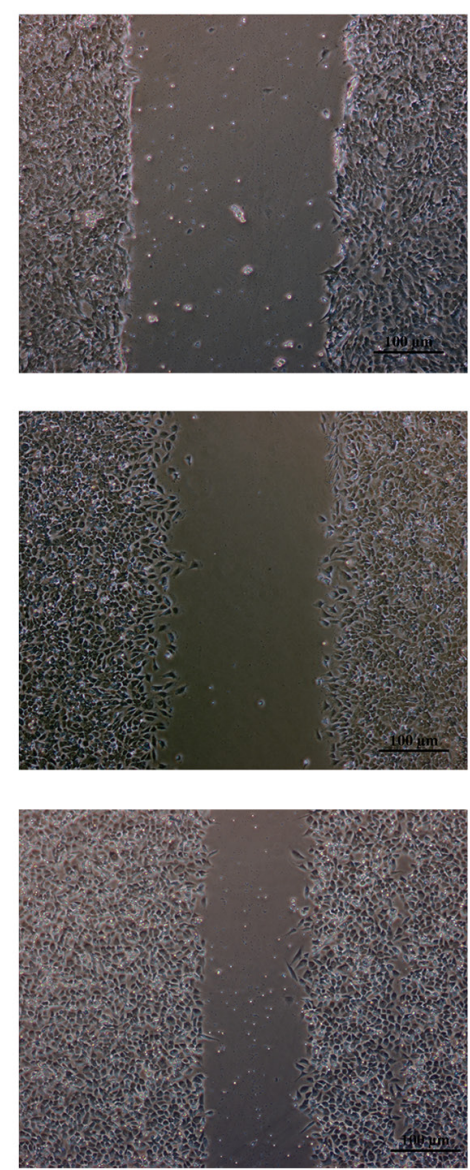

B

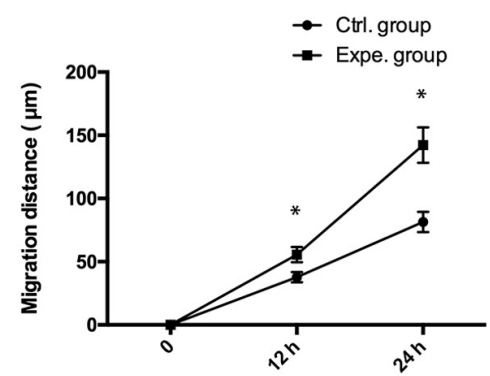

C

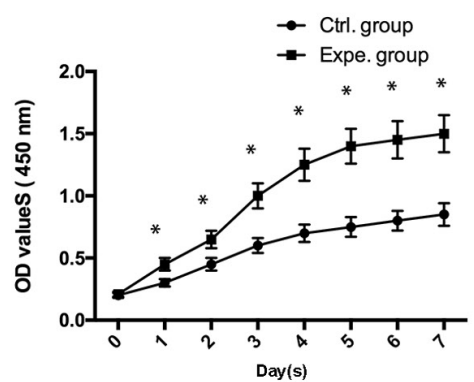

Figure 2. Proliferation and migration of human NP cells treated with exosomes. (A) Light microscopy images of the scratch wound assay (scale bar, $100 \mu \mathrm{m}$ ) and (B) quantified migration distance indicating that ADSC-derived exosomes significantly stimulated the migration rate of human NP cells. (C) The Cell Counting Kit-8 assay demonstrated that the proliferation rate of human NP cells grown with ADSC-derived exosomes was higher than that in the control group. " $\mathrm{P}<0.05$ vs. 0 h. NP, nucleus pulposus; ADSC, adipose-derived stem cell; Ctrl., control; Expe., experimental; OD, optical density.

proliferation was significantly increased in the presence of extracellular nanovesicles from days 1-7 (Fig. 2C).

ADSC-derived extracellular nanovesicles promote the chondrocytic gene expression in human NP cells. It was observed that ADSC-derived extracellular nanovesicles significantly promoted the mRNA expression of chondrocytic genes (collagen-II, aggrecan and Sox-9) in human NP cells in the experimental group compared with that in the control group $(\mathrm{P}<0.05)$ at day 7 (Fig. 3A), day 14 (Fig. 3B) and day 21 (Fig. 3C).

Telomerase activity in human NP cells. The effects of ADSC-derived extracellular nanovesicles on the telomerase activity in human NP cells were examined. Compared with that in the control group, a significant increase in telomerase activity was observed in human NP cells treated with extracellular nanovesicles in the experimental group $(\mathrm{P}<0.05$; Fig. 4).

Levels of inflammatory cytokines. Human NP cells were cultured with or without extracellular nanovesicles for 7 days. Inflammatory cytokine levels were measured by ELISAs. As indicated in Fig. 5, IL-1 $\alpha$, IL-1 $\beta$, IL-6, IL-17, NF-кB-p65 and
TNF- $\alpha$ were significantly reduced after 7 days culture with exosomes $(\mathrm{P}<0.05)$, indicating that the extracellular nanovesicles inhibited the secretion of inflammatory cytokines (Fig. 5).

\section{Discussion}

Emerging evidence has indicated that MSCs are promising options for the treatment of human intervertebral disc degeneration $(11,23)$. Cao et al $(24)$ reported that co-culture of BMSCs may be able to delay NP-cell matrix degeneration and co-culturing BMSCs with NP cells appeared to result in the promotion of the gene expression of aggrecan, collagen-II and Sox-9. Leung et al (25) demonstrated that MSCs are able to suppress abnormal deposition of collagen-I in the NP and modulate the profibrotic mediators matrix metalloproteinase-12 and heat shock protein 47 , thus reducing collagen aggregation and maintaining proper fibrillar properties and function. However, the mechanisms underlying the therapeutic effects of MSCs have remained elusive. A recent study indicated that the therapeutic action of MSCs is not dependent on the engraftment of MSCs at the site of injury or the differentiation capability of the transplanted MSCs, but rather the secretion of paracrine factors (26). 
A

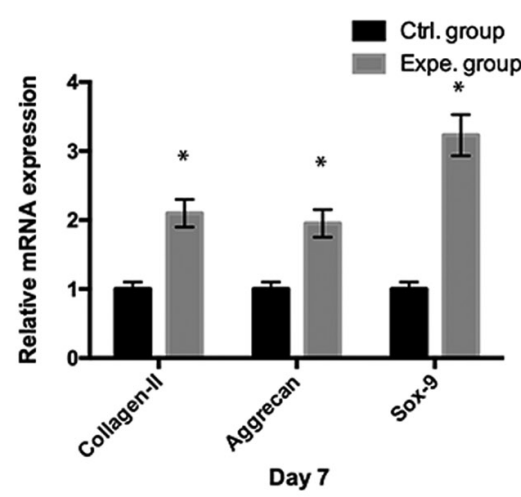

B

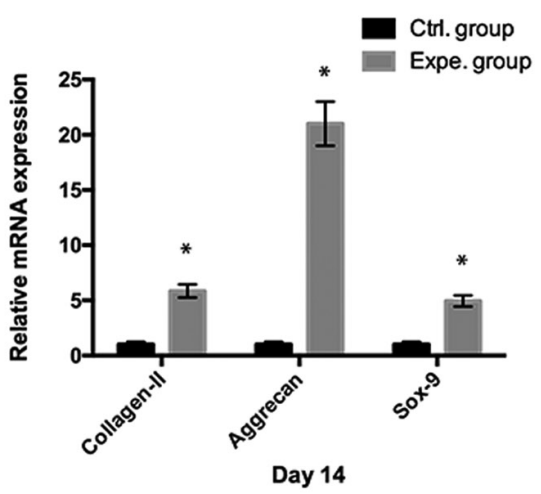

C

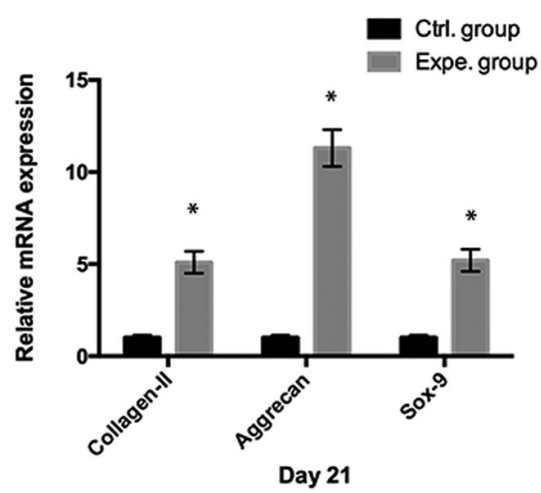

Figure 3. Chondrocytic gene expression of human NP cells. Analysis of the mRNA expression of collagen-II, aggrecan and Sox-9 suggested that exosome stimulation significantly induced chondrocytic gene expression at (A) day 7, (B) day 14 and (C) day 21 . * $<<0.05$ vs. Ctrl. group. NP, nucleus pulposus; Ctrl., control; Expe., experimental.

Ctrl. group

Expe.group

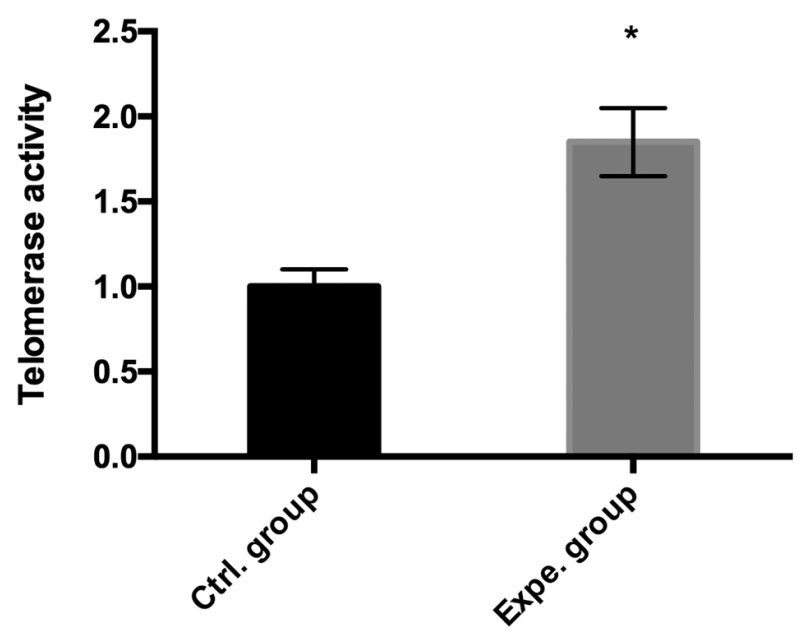

Figure 4. Telomerase activity. Detection of telomerase activity by PCR-ELISA in proteins from NP cells with or without exosomes, and the results indicated that exosomes stimulated the telomerase activity of human NP cells. ${ }^{*} \mathrm{P}<0.05$ vs. control group. NP, nucleus pulposus; Ctrl., control; Expe., experimental.

It has been reported that the therapeutic efficacy is attributed to the secretion of extracellular nanovesicles, secreted bilipid membrane vesicles of 40-100 nm in diameter that have the natural ability to transmit intercellular signals, such as carrying small RNAs, DNA and proteins (27). In the present study, the isolated ADSC-derived extracellular nanovesicles were characterized and transmission electron microscopy and laser diffraction demonstrated the cup-shaped morphology of the isolated extracellular nanovesicles with diameters of 40-100 $\mathrm{nm}$. NP cells have been reported to have a low capacity for proliferation and migration (28) and the effect of extracellular nanovesicles on the proliferation and migration of NP cells was evaluated in the present study. The results suggested that ADSC-derived extracellular nanovesicles improved the proliferation and migration activity of the degenerated
NP cells. Lu et al (29) reported that in their experiment, BM-MSC-derived exosomes promoted NP-cell proliferation.

Intervertebral disc degeneration is characterized by decreased expression of chondrogenic genes, such as aggrecan, collagen-II and Sox-9. Kim et al (30) demonstrated that co-culture with adipose-derived stem cells restored the chondrogenic properties of degenerative NP cells. In the present study, the expression levels of chondrogenic genes were significantly higher in extracellular nanovesicle-treated degenerative NP cells than those in the degenerative NP cells cultured alone, indicating that extracellular nanovesicles improved the viability of degenerative NP cells. This was further supported by the results for telomerase activity, which suggested that extracellular nanovesicles improved telomerase activity.

Intervertebral disc degeneration is a chronic degenerative disease characterized by back, neck and/or radicular pain (31). It is well known that increases in the levels of inflammatory cytokines secreted by intervertebral disc cells have a role in the occurrence of disc degeneration disease, such as IL-1 $\alpha$, IL-1 $\beta$, IL-6, IL-17, NF- $\kappa$ B-p65 and TNF- $\alpha$ (32). The release of these inflammatory cytokines stimulates chemokine production and extracellular matrix degradation and then causes degeneration of the intervertebral disc tissues, further resulting in lumbar disc herniation and radicular pain (33). Recent evidence has demonstrated that MSCs have great potential for application in the treatment of intervertebral disc degeneration, as MSCs have chondrogenic differentiation potential, but also have a vital role in immunoregulation and tissue repair/regeneration through the secretion of various soluble factors, such as extracellular nanovesicles (34). The present study indicated that ADSC-derived extracellular nanovesicle treatment decreased the secretion of inflammatory cytokines (IL-1 $\alpha$, IL-1 $\beta$, IL-6, IL-17, NF- $\kappa$ B-p65 and TNF- $\alpha$ ).

As a limitation to the present study, only one dose of extracellular vesicles was used when performing functional assays, as the quantity of extracellular vesicles that may be isolated from MSCs is low. Multiple doses of extracellular vesicles should be used when performing functional assays in future studies. 

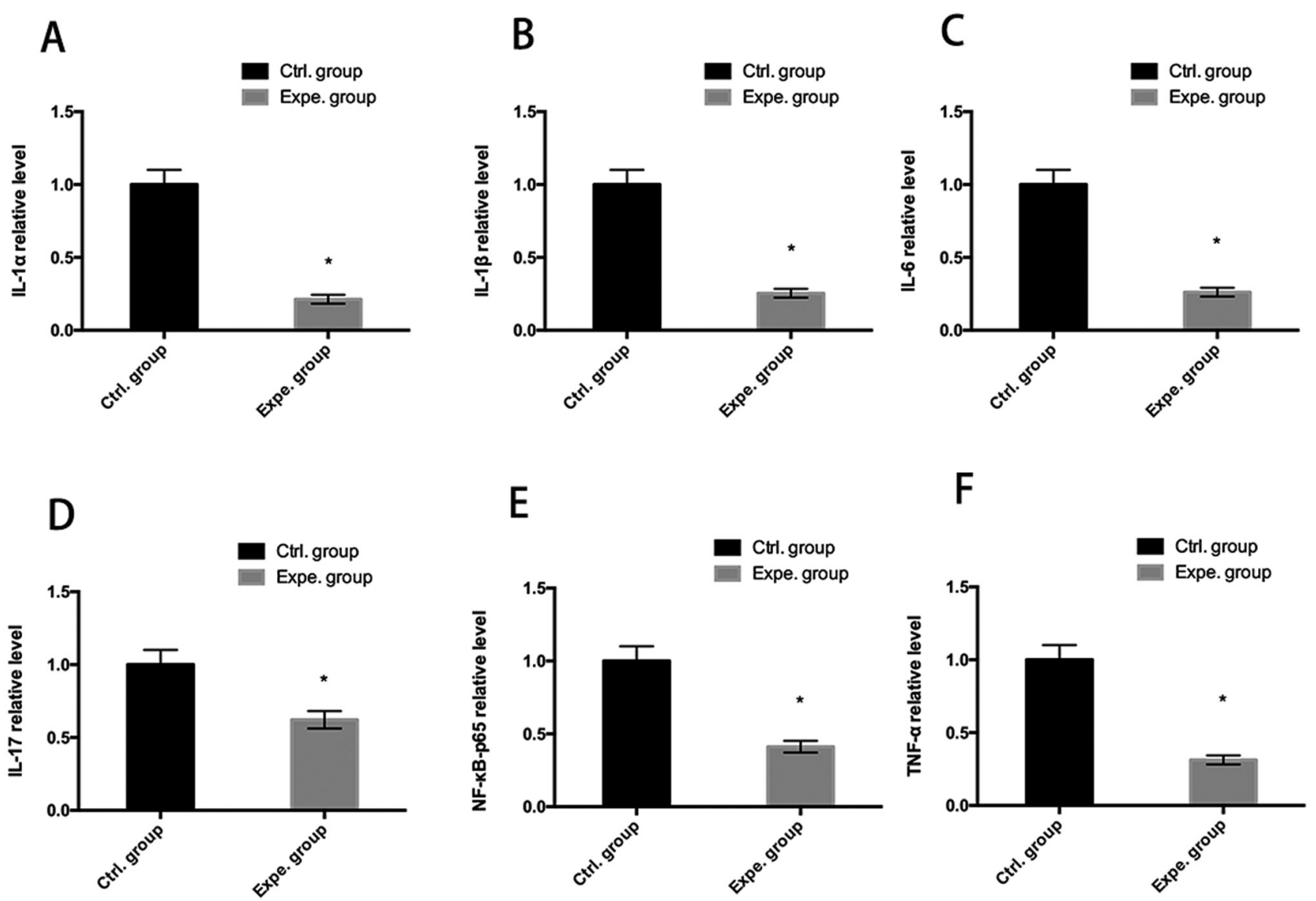

Figure 5. Analysis of inflammatory cytokines. A relative decrease in the inflammatory cytokines (A) IL-1 $\alpha$, (B) IL-1 $\beta$, (C) IL-6, (D) IL-17, (E) NF-кB-p65 and (F) TNF- $\alpha$ was determined at day 7 in the experimental group as compared to the control group. "P<0.05 vs. control group. Ctrl., control; Expe., experimental.

In conclusion, ADSC-derived extracellular nanovesicles improved the proliferation and migration of degenerative NP cells and inhibited inflammatory activity, which may provide a promising option for the treatment of intervertebral disc degeneration disease.

\section{Acknowledgements}

Not applicable.

\section{Funding}

This study was supported by the Jiangsu Provincial Medical Innovation Team (grant no. CXTDB2017004), the Natural Science Foundation of Yangzhou (grant no. YZ2017116) and Key Supporting Technical Projects by Norhern Jiangsu People's Hospital (grant no. fcjs202004).

\section{Availability of data and materials}

The datasets used and/or analyzed during the current study are available from the corresponding author on reasonable request.

\section{Authors' contributions}

ZZ contributed to the conception and design of the study, the acquisition, analysis and interpretation of the data of the study. XMF contributed to the acquisition, analysis and interpretation of data of the study. LZ contributed to the analysis and interpretation of data of the study. JY contributed to the analysis and interpretation of data of the study, and drafting of the work and its critical revision for important intellectual content. JH contributed to the conception and design of the study. JC contributed to the conception and design of the study. SZ contributed to the conception and design of the study, the acquisition, analysis and interpretation of the data of the study. QW contributed to the analysis and interpretation of data of the study. All authors contributed to the drafting of the work and its critical revision for important intellectual content, read and approved the final version of the manuscript and agreed to be accountable for all aspects of the study in ensuring that questions related to the accuracy or integrity of any part of the work are appropriately investigated and resolved. ZZ and QW confirm the authenticity of all the raw data.

\section{Ethics approval and consent to participate}

This study was approved by the Ethics Committee of Northern Jiangsu People's Hospital (Yangzhou, China) and informed consent was obtained from all patients.

\section{Patient consent for publication}

Not applicable. 


\section{Competing interests}

The authors declare that they have no competing interests.

\section{References}

1. Fernandez-Moure J, Moore CA, Kim K, Karim A, Smith K, Barbosa Z, Van Eps J, Rameshwar P and Weiner B: Novel therapeutic strategies for degenerative disc disease: Review of cell biology and intervertebral disc cell therapy. SAGE Open Med 6: $2050312118761674,2018$.

2. Petit A and Roquelaure Y: Low back pain, intervertebral disc and occupational diseases. Int J Occup Saf Ergon 21: 15-19, 2015.

3. Steelman T, Lewandowski L, Helgeson M, Wilson K, Olsen C and Gwinn D: Population-based risk factors for the development of degenerative disc disease. Clin Spine Surg 31: E409-E412, 2018.

4. Jakoi AM, Pannu G, D'Oro A, Buser Z, Pham MH, Patel NN Hsieh PC, Liu JC, Acosta FL, Hah R and Wang JC: The clinical correlations between diabetes, cigarette smoking and obesity on intervertebral degenerative disc disease of the lumbar spine. Asian Spine J 11: 337-347, 2017.

5. Teraguchi M, Yoshimura N, Hashizume H, Muraki S, Yamada H, Minamide A, Oka H, Ishimoto Y, Nagata K, Kagotani R, et al Prevalence and distribution of intervertebral disc degeneration over the entire spine in a population-based cohort: The wakayama spine study. Osteoarthritis Cartilage 22: 104-110, 2014.

6. Clouet J, Fusellier M, Camus A, Le Visage C and Guicheux J: Intervertebral disc regeneration: From cell therapy to the development of novel bioinspired endogenous repair strategies. Adv Drug Deliv Rev 146: 306-324, 2019.

7. Alvi MA, Kerezoudis P, Wahood W, Goyal A and Bydon M: Operative approaches for lumbar disc herniation: A systematic review and multiple treatment meta-analysis of conventional and minimally invasive surgeries. World Neurosurg 114: 391-407 e2, 2018.

8. Ayala-Cuellar AP, Kang JH, Jeung EB and Choi KC: Roles of mesenchymal stem cells in tissue regeneration and immunomodulation. Biomol Ther (Seoul) 27: 25-33, 2019.

9. Richardson SM, Kalamegam G,Pushparaj PN, Matta C, Memic A Khademhosseini A, Mobasheri R, Poletti FL, Hoyland JA and Mobasheri A: Mesenchymal stem cells in regenerative medicine: Focus on articular cartilage and intervertebral disc regeneration. Methods 99: 69-80, 2016.

10. Yang F, Leung VYL, Luk KDK, Chan D and Cheung KMC: Mesenchymal stem cells arrest intervertebral disc degeneration through chondrocytic differentiation and stimulation of endogenous cells. Mol Ther 17: 1959-1966, 2009.

11. Centeno C, Markle J, Dodson E, Stemper I, Williams CJ, Hyzy M, Ichim $\mathrm{T}$ and Freeman M: Treatment of lumbar degenerative disc disease-associated radicular pain with culture-expanded autologous mesenchymal stem cells: A pilot study on safety and efficacy. J Transl Med 15: 197, 2017.

12. Raik S, Kumar A and Bhattacharyya S: Insights into cell-free therapeutic approach: Role of stem cell 'soup-ernatant'. Biotechnol Appl Biochem 65: 104-118, 2018.

13. Liu X, Yang Y, Li Y, Niu X, Zhao B, Wang Y, Bao C, Xie Z, Lin $\mathrm{Q}$ and Zhu L: Integration of stem cell-derived exosomes with in situ hydrogel glue as a promising tissue patch for articular cartilage regeneration. Nanoscale 9: 4430-4438, 2017.

14. Merino-Gonzalez C, Zuñiga FA, Escudero C, Ormazabal V, Reyes C, Nova-Lamperti E, Salomón C and Aguayo C: Mesenchymal stem cell-derived extracellular vesicles promote angiogenesis: Potencial clinical application. Front Physiol 7: 24 2016.

15. Bucan V, Vaslaitis D, Peck CT, Strauß S, Vogt PM and Radtke C: Effect of exosomes from rat adipose-derived mesenchymal stem cells on neurite outgrowth and sciatic nerve regeneration after crush injury. Mol Neurobiol 56: 1812-1824, 2019.

16. Zhu Y, Wu Y, Cheng J, Wang Q, Li Z, Wang Y, Wang D, Wang H, Zhang W,Ye J, et al: Pharmacological activation of TAZ enhances osteogenic differentiation and bone formation of adipose-derived stem cells. Stem Cell Res Ther 9: 53, 2018
17. Chen D, Xia D, Pan Z, Xu D, Zhou Y, Wu Y, Cai N, Tang Q, Wang C, Yan M, et al: Metformin protects against apoptosis and senescence in nucleus pulposus cells and ameliorates disc degeneration in vivo. Cell Death Dis 7: e2441, 2016.

18. Baglio SR, Rooijers K, Koppers-Lalic D, Verweij FJ, Lanzón MP, Zini N, Naaijkens B, Perut F, Niessen HWM, Baldini N and Pegtel DM: Human bone marrow- and adipose-mesenchymal stem cells secrete exosomes enriched in distinctive miRNA and tRNA species. Stem Cell Res Ther 6: 127, 2015.

19. Qu Y, Zhang Q, Cai X, Li F, Ma Z, Xu M and Lu L: Exosomes derived from miR-181-5p-modified adipose-derived mesenchymal stem cells prevent liver fibrosis via autophagy activation. J Cell Mol Med 21: 2491-2502, 2017.

20. Zhang J, Guan J, Niu X, Hu G, Guo S, Li Q, Xie Z, Zhang C and Wang Y: Exosomes released from human induced pluripotent stem cells-derived MSCs facilitate cutaneous wound healing by promoting collagen synthesis and angiogenesis. J Transl Med 13: 49, 2015.

21. Barra GB, Santa Rita TH, Almeida ALSC, Jácomo RH and Nery LFA: Serum has higher proportion of janus kinase 2 V617F mutation compared to paired EDTA-whole blood sample: A model for somatic mutation quantification using QPCR and the $2^{-\Delta \Delta C q}$ method. Diagnostics (Basel) 10: 153, 2020.

22. Chung SS, Adekoya D, Enenmoh I, Clarke O, Wang P, Sarkyssian M, Wu Y and Vadgama JV: Salinomycin abolished STAT3 and STAT1 interactions and reduced telomerase activity in colorectal cancer cells. Anticancer Res 37: 445-453, 2017.

23. Perez-Cruet M, Beeravolu N, McKee C, Brougham J, Khan I, Bakshi S and Chaudhry GR: Potential of human nucleus pulposus-like cells derived from umbilical cord to treat degenerative disc disease. Neurosurgery 84: 272-283, 2019.

24. Cao C, Zou J, Liu X, Shapiro A, Moral M, Luo Z, Shi Q, Liu J, Yang $\mathrm{H}$ and Ebraheim N: Bone marrow mesenchymal stem cells slow intervertebral disc degeneration through the NF-kB pathway. Spine J 15: 530-538, 2015.

25. Leung VY, Aladin DMK, Lv F, Tam V, Sun Y, Lau RYC, Hung SC, Ngan AHW, Tang B, Lim CT, et al: Mesenchymal stem cells reduce intervertebral disc fibrosis and facilitate repair. Stem Cells 32: 2164-2177, 2014.

26. Lai RC, Tan SS, The BJ, Sze SK, Arslan F, de Kleijn DP, Choo A and Lim SK: Proteolytic potential of the MSC exosome proteome: Implications for an exosome-mediated delivery of therapeutic proteasome. Int J Proteomics 2012: 971907, 2012.

27. Vizoso FJ, Eiro N, Cid S, Schneider J and Perez-Fernandez R: Mesenchymal stem cell secretome: Toward cell-free therapeutic strategies in regenerative medicine. Int J Mol Sci 18: 1852, 2017.

28. Guillaume O, Naqvi SM, Lennon K and Buckley CT: Enhancing cell migration in shape-memory alginate-collagen composite scaffolds: In vitro and ex vivo assessment for intervertebral disc repair. J Biomater Appl 29: 1230-1246, 2015.

29. Lu K, Li HY, Yang K, Wu JL, Cai XW, Zhou Y and Li CQ: Exosomes as potential alternatives to stem cell therapy for intervertebral disc degeneration: In-vitro study on exosomes in interaction of nucleus pulposus cells and bone marrow mesenchymal stem cells. Stem Cell Res Ther 8: 108, 2017.

30. Kim JS, Kwon D, Cha BH, Moon BK, Jeong YG, Han IB, Park H and Lee SH: Restoration of chondrogenic properties of degenerative nucleus pulposus cells by repeated co-culture with adipose-derived stem cells. J Industrial Engineering Chem 60: 185-191, 2018.

31. Dowdell J, Erwin M, Choma T, Vaccaro A, Iatridis J and Cho SK: Intervertebral disk degeneration and repair. Neurosurgery 80 (Suppl 3): S46-S54, 2017

32. Khan AN, Jacobsen HE, Khan J, Filippi CG, Levine M, Lehman RA Jr, Riew KD, Lenke LG and Chahine NO: Inflammatory biomarkers of low back pain and disc degeneration: A review. Ann N Y Acad Sci 1410: 68-84, 2017.

33. Risbud MV and Shapiro IM: Role of cytokines in intervertebral disc degeneration: Pain and disc content. Nat Rev Rheumatol 10: 44-56, 2014.

34. Lai RC, Yeo RW and Lim SK: Mesenchymal stem cell exosomes. Semin Cell Dev Biol 40: 82-88, 2015. 